\title{
Argan oil prevents prothrombotic complications by lowering lipid levels and platelet aggregation, enhancing oxidative status in dyslipidemic patients from the area of Rabat (Morocco)
}

\author{
Adil Haimeur ${ }^{1,2}$, Hafida Messaouri², Lionel Ulmann'1, Virginie Mimouni ${ }^{1}$, Azelarab Masrar ${ }^{3}$, Abdelmjid Chraibi ${ }^{4}$,
} Gérard Tremblin ${ }^{1}$ and Nadia Meskini ${ }^{2 *}$

\begin{abstract}
Background: It is now established that patients with hyperlipidemia have a high risk of atherosclerosis and thrombotic complications, which are two important events responsible for the onset and progression of cardiovascular disease. In the context of managing dyslipidemia by means of dietary advice based on the consumption of argan oil, we wanted to investigate the effect of virgin argan oil on plasma lipids, and for the first time, on the platelet hyperactivation and oxidative status associated with dyslipidemia. This study concerns patients recruited in the area of Rabat in Morocco.

Methods: 39 dyslipidemic (79\% women) patients were recruited for our study in the area of Rabat in Morocco. They were randomly assigned to the two following groups: the argan group, in which the subjects consumed $25 \mathrm{~mL} /$ day of argan oil at breakfast for 3 weeks, and the control group in which argan oil was replaced by butter.

Results: After a 3-week consumption period, blood total cholesterol was significantly lower in the argan oil group, as was LDL cholesterol (23.8\% and 25.6\% lower, respectively). However, the HDL cholesterol level had increased by $26 \%$ at the end of the intervention period compared to baseline. Interestingly, in the argan oil group thrombin-induced platelet aggregation was lower, and oxidative status was enhanced as a result of lower platelet MDA and higher GPx activity, respectively.
\end{abstract}

Conclusions: In conclusion, our results, even if it is not representative of the Moroccan population, show that argan oil can prevent the prothrombotic complications associated with dyslipidemia, which are a major risk factor for cardiovascular disease.

Keywords: Platelet aggregation, Argan oil, Dyslipidemia, Oxidative stress

\section{Background}

Cardiovascular disease (CVD) is the main cause of increasing mortality worldwide. In 2008, heart disease accounted for $23 \%$ of deaths in Morocco [1]. Dyslipidemia, the main risk factor for CVD, is a heterogeneous metabolic disorder involving multiple etiologies; it is commonly characterized by increased triglycerides, low-

\footnotetext{
*Correspondence: nadia_meskini@hotmail.com

${ }^{2}$ Université Hassan II, Equipe Nutrition, Environnement, Santé. Laboratoire de Virologie, Microbilogie, Qualité/Ecotoxicologie et Biodiversité, Faculté des Sciences et Techniques, Mohammedia, Morocco

Full list of author information is available at the end of the article
}

density lipoprotein (LDL) cholesterol and apolipoprotein B (apo-B) levels, and reduced plasma high-density lipoprotein (HDL) cholesterol [2]. These components of dyslipidemia confer an increased risk for atherosclerosis and thrombosis, which are two important events responsible for the onset and progression of CVD [3]. It is now established that patients with hyperlipidemia have a high risk of atherosclerosis and thrombotic complications [4-6]. Several mechanisms have been suggested by which alterations in plasma lipid levels may promote atherosclerosis [7-9]. One major determinant of the prothrombotic state associated with hypercholesterolemia appears to be related

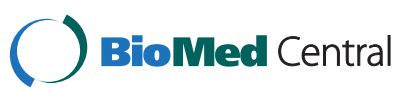


to enhanced platelet activation. Indeed, platelets are known to play a fundamental role in atherogenesis and in the pathology of atherothrombotic disorders [10]. In-vivo platelet activation has been reported in type-IIa hypercholesterolemic patients [11], suggesting that high levels of oxidized LDL, via changes in the composition of platelet membrane phospholipids and cholesterol, may increase the platelet reactivity associated with enhanced thromboxane $\mathrm{A}_{2}\left(\mathrm{TxA}_{2}\right)$ biosynthesis. The involvement of blood platelet activation in the onset of atherosclerosis is well established. It has long been recognized that platelets are involved in the late thrombotic complications of atherosclerosis plaque due to erosion of the endothelium or rupture of the fibrous cap that covers the lipid core within the plaque [12]. The association of platelet activation with acute CVD justifies anti-platelet therapy as a standard treatment for those at high risk of atherothrombosis.

Several epidemiological, clinical, and experimental studies have established that diet can have beneficial effects on the cardiovascular system, and can therefore be considered to provide an effective therapeutic tool for preventing several CVD risk factors, such as dyslipidemia, platelet hyper-reactivity, and diabetes [13,14]. As far as ingested lipids are concerned, the influence of their quality and quantity has often been demonstrated in human and animal models [15]. Haimeur et al. [16] showed that a high fat diet consumption increased the incidence of CVD in rats, by increasing blood lipids level, platelet aggregation and hepatic oxidative stress, however these risk factors of CVD were lower in rats fed with a high fat diet supplemented with a freeze-dried of Odontella aurita, a marine diatom rich in omega-3 (eicosapentaenoic acid) and antioxidants molecules.

Argan oil is a natural vegetable oil that has been widely used in both the daily diet and folk medicine in Morocco. It is extracted from the kernels of Argania spinosa, an endemic tree growing in the south-western region of Morocco. The main traditional use of this edible oil is for nutritional purposes by consuming it directly on toast, generally during breakfast [17]. This oil is rich in unsaturated fatty acids (80\%) principally oleic and linoleic acids (44.8\% and $33.7 \%$, respectively) [18]. Interestingly, the unsaponifiable fraction of argan oil (1\% of the oil constituents) contains mainly antioxidant compounds, such as tocopherols, especially $\gamma$-tocopherol, which are present in higher portions than in olive oil (637 $\mathrm{mg} / \mathrm{kg}$ versus $258 \mathrm{mg} / \mathrm{kg}$ of total tocopherols, respectively) (Table 1) [18]. As could be expected from its interesting composition, argan oil has demonstrated its pharmacological effects in several studies. Initially demonstrated in rats [19-22], the hypocholesterolemic potency of argan oil in humans has also been demonstrated by a cohort study of 60 healthy men [23]. Numerous nutritional studies have also shown that argan oil lowers LDL
Table 1 Chemical composition of argan oil [18]

\begin{tabular}{ll}
\hline Fatty acids & (\%) \\
\hline C 16:0 & 13.4 \\
C 18:0 & 5.1 \\
C 18:1 & 44.8 \\
C 18:2 & 35.7 \\
C 18:3 & 0.1 \\
Sterols $\mathbf{m g / 1 0 0 ~ g ~ o i l ~}$ & \\
Schottenol & 142 \\
Spinasterol & 115 \\
Stigmasta-8,22-dien-3-ol & 9 \\
Others & 29 \\
Tocopherols $\mathbf{m g / K g}$ oil & \\
a-tocopherol & 35 \\
S-tocopherol & 122 \\
Y-tocopherol & 480 \\
Phenols $\boldsymbol{\mu g} / \mathbf{K g}$ oil & \\
Vanillic acid & 67 \\
Syringic acid & 37 \\
Ferulic acid & 3147 \\
Tyrosol & 12 \\
\hline
\end{tabular}

cholesterol and has antioxidant properties; these properties have been demonstrated in a cohort study involving 96 people [24]. Here, the subjects who consumed argan oil on a regular basis presented with significantly lower levels of plasma LDL cholesterol and lipoprotein A Lp (a), and lower levels of plasma lipoperoxides (thiobarbituric acid reactive substances, TBARS) [24]. Argan oil also increases HDL cholesterol and lowers triglyceride levels in men [25], and therefore regular argan oil consumption could potentially prevent diabetes [26]. Furthermore, when rats were fed for 4 weeks with $10 \mathrm{~mL} / \mathrm{kg} /$ day of argan oil, the thrombin-induced aggregation of isolated platelets was significantly inhibited (36\%), however, the tail bleeding time remained unchanged [27]. Recently, Mekhfi et al. [28] have reported that argan oil has antithrombotic activity in rats, which could be related to its antiplatelet rather than its anticoagulant activity.

Argan oil has already been reported to be a hypolipidemic factor in both human and animal models without producing any adverse effects [20,24]. However, the anti-aggregant effect of argan oil has so far only been described in-vitro in rats [27]. Thus the originality of the study reported here, is investigating the protective effect of argan oil against platelet disorders, such as hyperactivity and oxidative stress in dyslipidemic patients. According to the low sampling of patient recruited in the area of the city of Rabat, our exploratory study is not significant of the moroccan population. 


\section{Methods \\ Patients}

This study was conducted on dyslipidemic patients attending the Endocrinology Department of the Ibn Sina University Hospital, Rabat, Morocco. The inclusion criteria was patients with hypercholesterolemia or/and hypertriglyceridemia. The exclusion criteria included myocardial infarction, the presence of hepatic or renal disease, the use of anti-aggregant drugs (aspirin, thienopyridines) or of lipidlowering drugs (statins), unusual diet (low or high fat or carbohydrate diets), or taking hormone therapy during the previous 6 months. The protocol and objectives of this study were explained to the participants in detail. Before the recruitment, patients give their consent orally. The study protocol was approved by the Institutional Ethics Committee of the Endocrinology, Diabetology, and $\mathrm{Nu}$ trition Department, Ibn Sina University Hospital, Rabat, Morocco. Among the 78 patients who consulted, a total of 39 dyslipidemic patients were selected on the basis of the inclusion criteria. All the patients included underwent a thorough physical examination in the Service at 3 consultations during the study.

\section{Study design}

This study was conducted according to the protocol described by Derouiche et al. [25]. The study design consisted of two diet periods. The first diet period (stabilization period) lasted 2 weeks during which all the patients consumed $20 \mathrm{~g} /$ day of butter on toasted bread for breakfast, and both argan oil and olive oil were eliminated from their diet. This stabilization period was intended to ensure that all the patients had a similar fat intake and stable dyslipidemia parameters before starting the study. In the second diet period (nutritional intervention), which lasted 3 weeks, the patients were randomly assigned to one of two groups: the first group (18 patients) received the $20 \mathrm{~g} /$ day butter diet (control group: C); the second group (21 patients) received $25 \mathrm{~mL} /$ day of virgin argan oil instead of the butter with the toasted bread for breakfast (argan oil group: $\mathrm{AO})$. The daily eating habits of all the patients were investigated by means of a comprehensive food questionnaire covering all foods commonly consumed in Morocco. During the nutritional intervention period, all patients followed a lifestyle and diet recommended by the guidelines for the management of dyslipidemia [29]. The argan oil supplied to the patients was all purchased from the same source, and was extracted by an industrial process [30].

\section{Blood collection}

During the study, the patients provided 3 blood samples. The first was collected intravenously in vacuum tubes after they had fasted for $12 \mathrm{~h}$, and was used to confirm their dyslipidemia by serum lipid analysis. At the end of the stabilization period, the second sample was collected intravenously into two tubes: the first was a vacuum tube containing EDTA, and this blood was used for serum lipid analysis, and the other was a tube containing citric phosphate dextrose (CPD) anticoagulant (citric acid: $15.6 \mathrm{mM}$; sodium citrate: $89.4 \mathrm{mM}$, monosodium phosphate: 16.1 $\mathrm{mM}$ and dextrose: $128.7 \mathrm{mM}$ ) in a ratio of $1 / 7 \mathrm{v} / \mathrm{v} \mathrm{CPD} /$ blood, and this was used for platelet analysis (platelet aggregation test, basal $\mathrm{TxB}_{2}$ determination and platelet oxidative status). The third blood sample was similar to the second and was collected at the end of the nutritional intervention.

\section{Anthropometric and serum lipid parameters}

Demographic and anthropometric parameters (age, sex, weight, body mass index (BMI), blood pressure) were recorded for the patients at baseline. Serum total cholesterol (TC) and HDL-cholesterol levels were determined using commercial enzymatic kits (Randox cholesterol enzymatic kit and Randox HDL cholesterol precipitant for cholesterol and HDL-cholesterol, respectively, Crumlin, Co. Antrim, UK) according to Richmond's colorimetric procedure [31]. Serum triglyceride levels were determined using a commercial kit (Randox, Triglyceride Enzymatic Kit, Crumlin, Co. Antrim, UK) according to Trinder's colorimetric method [32]. The LDL-cholesterol level was calculated by Friedewald's formula [33]. LDL cholesterol $(\mathrm{g} / \mathrm{L})=$ total cholesterol $(\mathrm{g} / \mathrm{L})$ - HDL cholesterol $(\mathrm{g} / \mathrm{L})$ - triglycerides $/ 5(\mathrm{~g} / \mathrm{L})$.

\section{Platelet aggregation}

Platelets were isolated as previously described by Lagarde et al. [34]. Briefly, blood was centrifuged at $200 \mathrm{x}$ g for $15 \mathrm{~min}$ at $20^{\circ} \mathrm{C}$ to obtain platelet-rich plasma (PRP). PRP was then acidified to $\mathrm{pH} 6.4$ with $0.15 \mathrm{M}$ citric acid, and immediately centrifuged at $900 \mathrm{x} \mathrm{g}$ for $10 \mathrm{~min}$ at $20^{\circ} \mathrm{C}$. Platelet pellets were resuspended in Tyrode/HEPES buffer solution $(137 \mathrm{mM} \mathrm{NaCl}, 2.7 \mathrm{mM} \mathrm{KCl}, 11.9 \mathrm{mM} \mathrm{NaHCO}$, $0.41 \mathrm{mM} \mathrm{NaH} \mathrm{PO}_{4}, 1 \mathrm{mM} \mathrm{MgCl}, 5.5 \mathrm{mM}$ glucose, $5.0 \mathrm{mM}$ HEPES, pH 7.35). Platelet concentration was adjusted to $300000 / \mu \mathrm{L}$ before aggregation assay. The isolated platelets were then used to determine platelet aggregation by turbidimetry [35] using a PACKS 4 aggregometer (HELENA, TX, USA). Aggregation tests were performed at $37^{\circ} \mathrm{C}$ in cuvettes stirred at $1000 \mathrm{rpm}$. Isolated platelets were stimulated by exposure to thrombin (Sigma-Aldrich, SaintQuentin Fallavier, France) $0.2 \mathrm{U} / \mathrm{mL}$. The light transmission was recorded for $3 \mathrm{~min}$ after platelet stimulation. The platelet aggregation was quantified from the maximum change in light transmission through a washed platelet suspension, expressed as a percentage of the light transmission through the blank (Tyrode buffer).

\section{Platelet MDA measurement}

Platelet lipid peroxidation was evaluated by measuring the malondialdehyde (MDA) level according to the method 
described by Ohkawa [36]. To $500 \mu \mathrm{L}$ of platelet solution were added $100 \mu \mathrm{L}$ of $8.1 \%$ sodium dodecyl sulfate (SDS), $750 \mu \mathrm{L}$ of acetic acid $20 \%$ at $\mathrm{pH} 3.5,750 \mu \mathrm{L}$ of thiobarbituric acid (TBA) $0.8 \%$, and distilled water to make up the volume to $2.5 \mathrm{~mL}$. The tubes were maintained in a water bath at $95^{\circ} \mathrm{C}$ for $60 \mathrm{~min}$. The tubes were then immediately cooled in ice. $500 \mu \mathrm{L}$ of distilled water and $2.5 \mathrm{~mL}$ of a solution of $n$-butanol and pyridine $(15: 1 \mathrm{v} / \mathrm{v})$ were then added to each tube. The tubes were shaken vigorously before being centrifuged at $1000 \mathrm{x} g$ for $10 \mathrm{~min}$. The organic phase, corresponding to the upper layer, was aspirated and its color intensity measured at $532 \mathrm{~nm}$. A standard range was prepared using a 1,3,3,3- tetramethoxypropane (Sigma-Aldrich, Saint-Quentin Fallavier, France) solution.

\section{Platelet glutathione peroxidase activity}

The glutathione peroxidase (GPx) activity was determined in isolated platelets according to the Paglia and Valentine method [37], as modified by Chaudiére and Gérard [38]. Briefly, $100 \mu \mathrm{L}$ of platelet lysate was added to a final volume of $1.5 \mathrm{~mL}$ of a solution containing Tris- $\mathrm{HCl} / \mathrm{EDTA}$ (50 mM/0.1 mM) pH 7.6, NADPH, $\mathrm{H}^{+}(0.14 \mathrm{mM})$, reduced glutathione (GSH) (2 mM) and $0.7 \mathrm{U} / \mathrm{mL}$ of GSSGreductase. The mixture was incubated at $37^{\circ} \mathrm{C}$ for $3 \mathrm{~min}$. The reaction was started by adding $50 \mu \mathrm{L}$ tertiary butyl hydroperoxide $(t-\mathrm{BH})(0.2 \mathrm{mM})$. The change in absorbance was recorded at $340 \mathrm{~nm}$ for $5 \mathrm{~min}$ at $5 \mathrm{~s}$ intervals. An appropriate control without the platelet sample was run simultaneously. GPx activity was expressed in nmoles of hydroperoxide reduced per min and per mg protein.

\section{Thromboxane $\mathrm{B}_{2}$ measurement}

To determine the platelet thromboxane $\mathrm{B}_{2}\left(\mathrm{TxB}_{2}\right)$ level, isolated platelets were subjected to three successive freeze/thaw cycles to release the cell contents. The baseline $\mathrm{TxB}_{2}$ level was measured by enzyme immunoassay (EIA) Kit (Enzo-Life Sciences, Exeter, U.K) according to the Manufacturer's instructions.

\section{Assay of proteins}

Platelet total proteins were determined by the Bradford colorimetric method [39] using Biorad reagent and bovine serum albumin as standard (Sigma-Aldrich, SaintQuentin Fallavier, France).

\section{Statistical analysis}

All values were expressed as the mean \pm SD. After analysis of variance, the mean values were compared using Fisher's least significant difference test (Statgraphics Plus 5.1, Manugistics Inc., Rockville, MD, USA). P 0.05 was accepted for significant differences.

\section{Results}

\section{Baseline anthropometric parameters}

The baseline characteristics of the patients, including their sex and age as well as the risk factors associated with atherosclerosis diseases, are presented in Table 2. At the beginning of the intervention period, the data showed that both groups displayed abnormal blood lipid markers characterized by hypercholesterolemia and hypertriglyceridemia. According to the International Diabetes Federation (IDF) definition [40], all of the patients exhibited at least two components of metabolic syndrome (MS). In addition to hypertriglyceridemia and hypercholesterolemia, the most frequent component of the MS in our patients was central obesity.

\section{Serum lipid parameters}

After the three weeks of the nutritional intervention period, a significant improvement in atherogenic lipid parameters was found in the $\mathrm{AO}$ group patients as compared to the values found before the nutritional intervention period (Figure 1). The data showed significantly $(p=0.04)$ lower total cholesterol and LDL-cholesterol levels ( $p=$ $0.02)$ and significantly $(p=0.01)$ higher HDL-cholesterol levels in the group consuming argan oil than in the control group of patients (23.8\% versus $13.5 \%$, $25.6 \%$ versus $10.4 \%$, and $26 \%$ versus $6.6 \%$, respectively). However, there was no difference in triglyceride levels between the two groups after the three weeks of the intervention period.

\section{Platelet aggregation and $\mathrm{TxB}_{2}$ production}

Platelet hyperactivity is associated with an increase risk of adverse cardiovascular outcomes. We evaluated the platelet function of patients by studying the platelet response to aggregation agonist stimulation. The thrombin-induced aggregation exemple is shown in the Figure 2. The results obtained after the three weeks of the nutritional intervention period showed that the percentage thrombin-induced platelet aggregation was significantly $(p=0.03)$ lower in

\section{Table 2 Baseline anthropometrical and biological} characteristics of the study groups

\begin{tabular}{lcc}
\hline Characteristic & Control group & Argan oil group \\
\hline Sex (W/M) & $14 / 4$ & $17 / 4$ \\
Age (years) & $51 \pm 9$ & $55 \pm 8$ \\
Weight (Kg) & $74.9 \pm 13.0$ & $72.3 \pm 9.4$ \\
SBP (mmHg) & $11.9 \pm 1.3$ & $12.4 \pm 0.9$ \\
DBP (mmHg) & $6.7 \pm 0.9$ & $6.9 \pm 0.7$ \\
BMI (Kg/m2) & $29.6 \pm 5.0$ & $29.7 \pm 4.9$ \\
TC (g/L) & $2.53 \pm 0.3$ & $2.41 \pm 0.4$ \\
TG (g/L) & $1.62 \pm 0.1$ & $1.73 \pm 0.2$ \\
HDL-chl (g/L) & $0.45 \pm 0.07$ & $0.50 \pm 0.05$ \\
LDL-chl (g/L) & $1.13 \pm 0.2$ & $1.35 \pm 0.3$ \\
\hline
\end{tabular}




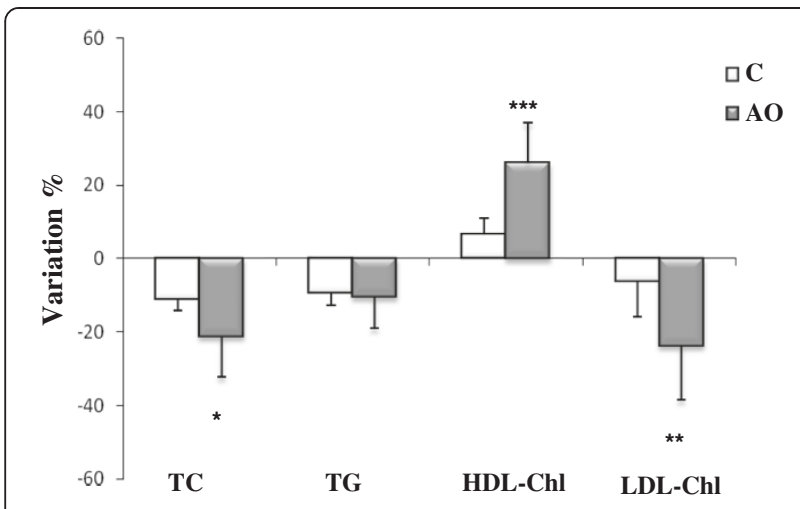

Figure 1 Variations in different plasma lipid markers after 3 weeks of nutritional intervention with argan oil. Data are the percentage variation of the lipid parameters between the two periods. Comparisons are made between the variation in the two groups of patients (C: control group; AO: argan oil group). TC: total cholesterol; TG: triacylglycerol ; HDL-Chl : high density lipoprotein cholesterol; LDL-Chl: low density lipoprotein cholesterol. After a one-way ANOVA, Student-Newman-Keuls multiple test, a comparison was made between the two groups of patients. ${ }^{* * *}: p=0.01 ; * *: p=0.02 ; *: p=0.04$.

the $\mathrm{AO}$ group than in the control group (44.5\% versus $62.6 \%$ ) at the end of the intervention period (Figure 3).

On another hand, the baseline concentration of $\mathrm{TxB}_{2}$, the stable catabolite of $\mathrm{TxA}_{2}$, tended to be lower in the AO group than in the control group after the three weeks of the intervention period, but this difference was not statistically significant $(p=0.1)$ (Figure 4$)$.

\section{Platelet oxidative status}

The results for the platelet oxidative status are shown in Figures 5 and 6 . After the three weeks of the intervention period, the data showed that platelet lipid peroxidation levels were lower in patients consuming argan oil, with an MDA level about $34 \%$ lower $(p=0.02)$ than in the control group at the end of the intervention period (Figure 5). This result was correlated with the activity of platelet GPx, the main enzyme catalyzing the reduction of lipid hydroperoxides to the corresponding alcohols (Figure 6). Interestingly, at the end of the intervention period, the platelet GPx activity in the AO group increased by $22 \%$ $(p=0.02)$ when compared to the control group.

\section{Discussion}

Hypercholesterolemia and platelet hyperactivity are associated with an increased risk of adverse cardiovascular outcomes (coronary artery disease, hypertension). Argan oil is rich in unsaturated fatty acids, and also contains a non-glyceride fraction characterized by the presence of sterols and antioxidant components, which gives it interesting biological and pharmacological properties. The present investigation was undertaken to assess the potential platelet anti-aggregant and antioxidant effects of argan oil on dyslipidemic patients. This clinical study involved evaluating the effects of argan oil after a three-week period during which consuming argan oil on toasted bread for breakfast was compared with consuming butter on toasted bread.

Interestingly, our results showed that argan oil appeared to be effective in preventing hypercholesterolemia in dyslipidemic patients. They show that after three weeks of argan oil consumption, circulating total cholesterol and LDL-cholesterol lowered by about $24 \%$ and $25 \%$, respectively, compared to the values obtained before the nutrition intervention period. In addition, patients who consumed

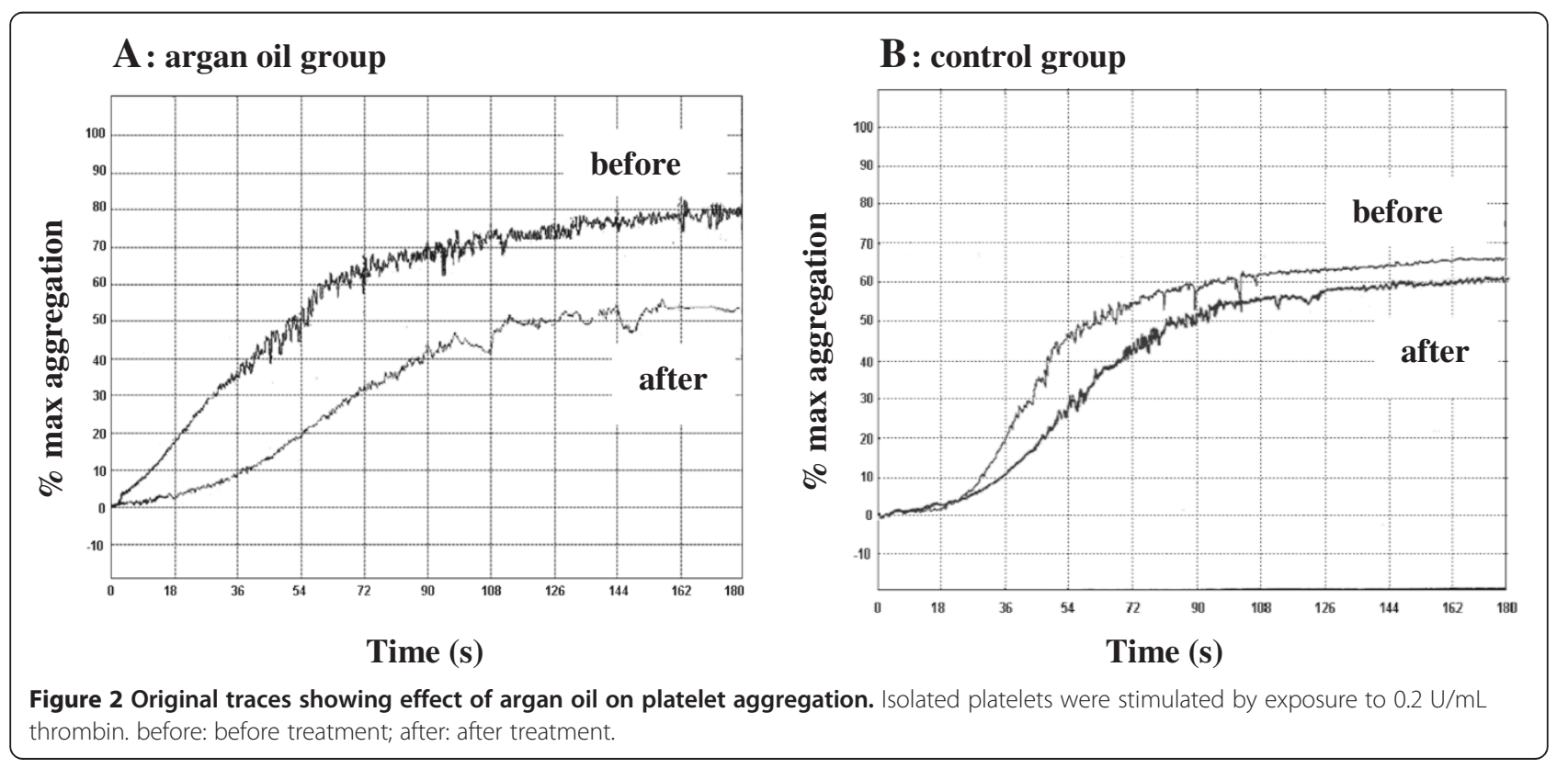




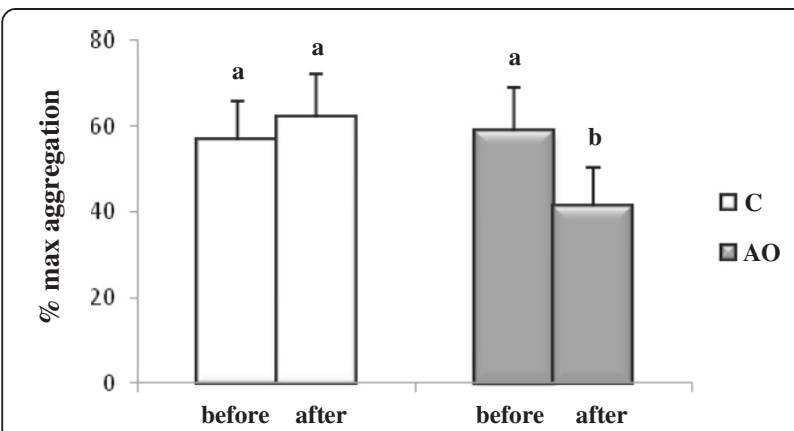

Figure 3 Platelet aggregation after 3 weeks of nutritional intervention with argan oil. Washed platelets were stimulated with thrombin $(0.2 \mathrm{U} / \mathrm{ml})$. Comparisons are made between the \% platelet aggregation before and after the intervention period in the two groups. C: control group; AO: argan oil group. After a one-way ANOVA, Student-Newman-Keuls multiple comparison test, results are arranged in increasing order from left to right $a>b(p<0.05)$.

argan oil had a higher plasma HDL-cholesterol levels than control patients. However, argan oil had no effect on circulating triglyceride levels after three weeks of intervention, whereas Derouiche et al. [25] reported that plasma triglyceride levels decreased significantly by $17.5 \%$ in healthy people consuming about $28 \mathrm{~mL} /$ day of argan oil over a 3-week period. Indeed, a lipid-lowering effect of argan oil has been reported in several other studies involving either animals or healthy male subjects [41-43], but our results demonstrate for the first time the hypocholesterolemic effect of argan oil on dyslipidemic patients. There is now abundant evidence that diets high in unsaturated fatty acids have a hypocholesterolemic effect when substituted for diets high in saturated fatty acids $[44,45]$. This has been showed by the beneficial effects of the "Mediterranean diet", which is attributed, besides the effects of fibers, complex carbohydrates, vegetable proteins

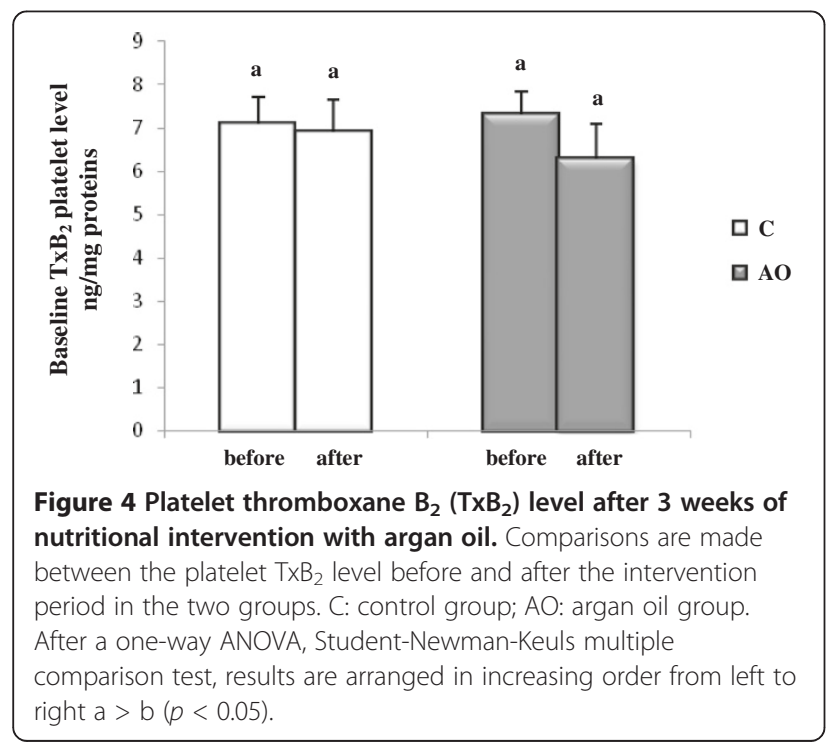

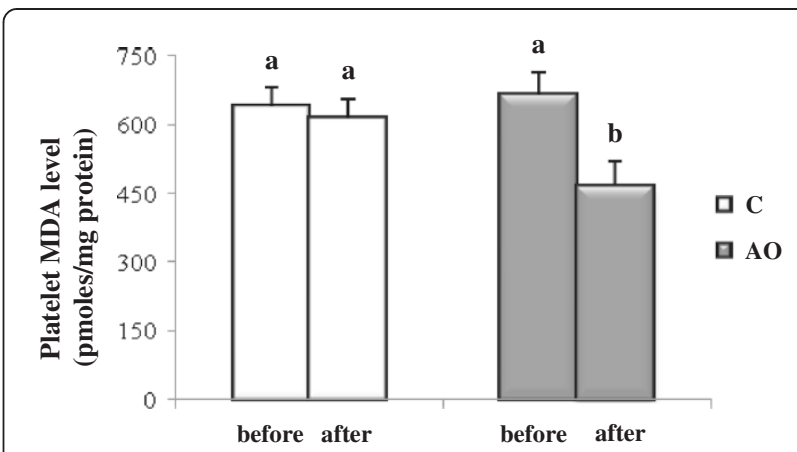

Figure 5 Platelet malondialdehyde (MDA) level after 3 weeks of nutritional intervention with argan oil. Comparisons are made between the platelet MDA level before and after the intervention period in the two groups. C: control group; AO: argan oil group. After a one-way ANOVA, Student-Newman-Keuls multiple comparison test, results are arranged in increasing order from left to right $a>b(p<0.05)$

etc, to its high content of olive oil, which is an important source of monounsaturated fatty acids (MUFAs) (73\% of oleic acid), whereas sunflower oil has a high content of polyunsaturated fatty acids (PUFAs) (61.6\% of linoleic acid) [46]. However, argan oil is also characterized by containing balanced proportions of MUFAs and PUFAs (44.8\% oleic acid and $33.7 \%$ linoleic acid) [18]. Linoleic acid (C18: $2 n-6)$ is an essential fatty acid, and is a precursor in the biosynthesis of arachidonic acid (C20: $4 n-$ 6). Arachidonic-derived fatty acids, particularly $\gamma$-linolenic acid, are known to reduce the total cholesterol, VLDL, and LDL-cholesterol levels in human and rat serum [47,48]. In addition to unsaturated fatty acids, other minor components of argan oil, such as plant sterols, may also be implicated in the hypocholesterolemic effect of this oil. Phytosterols differ from cholesterol by having ethyl or

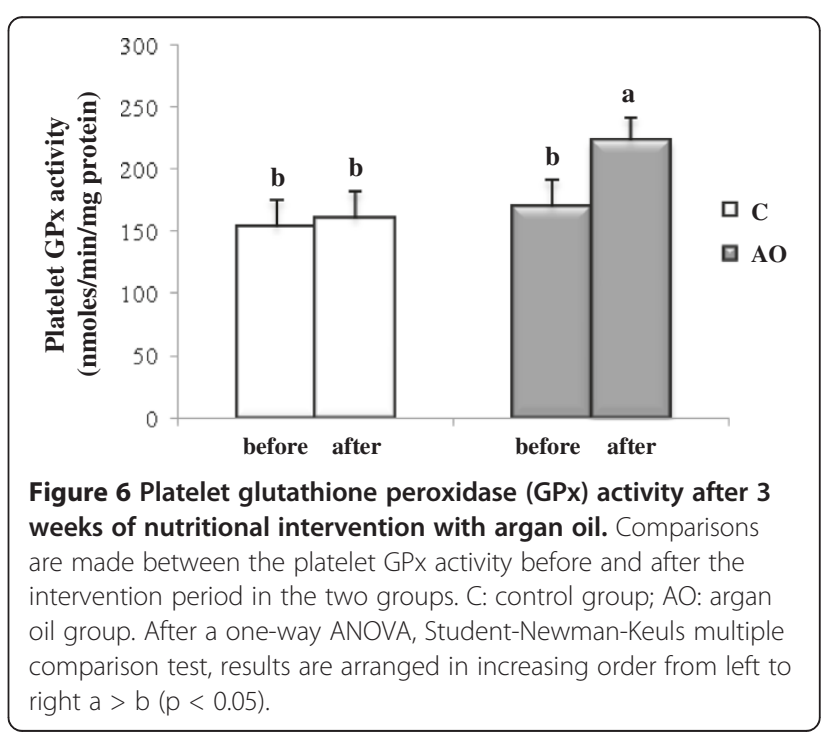


methyl groups at carbon-24 and/or a double bond at carbon-22. These features increase their hydrophobicity and reduce their absorption [49]. Phytosterols compete with endogenous cholesterol for space within bile salt micelles in the intestinal lumen, thereby reducing cholesterol absorption [50,51]. A recent, randomized, placebo-controlled, crossover trial reported that incorporating softgel capsules containing esterified plant sterols/stanols $1.8 \mathrm{~g} /$ day into the National Cholesterol Education Program (NCEP) Therapeutic Lifestyle Changes diet, produced favorable changes in atherogenic lipoprotein and cholesterol levels in subjects with hypercholesterolemia [52]. These results corroborate those found in several studies conducted with argan oil [25,41-43]. Furthermore, the cholesterol-lowering effect of argan oil observed in our study could be related to a synergistic effect of unsaturated fatty acids and sterols, the main constituents of this oil.

Accumulating evidence indicates that oxidative stress plays a significant role in the onset of atherosclerosis [53], and that platelet activation is an important contributing factor in the process of atherosclerosis and its thrombotic complications. In this study, we investigated the effect of argan oil on platelet aggregation, $\mathrm{TxB}_{2}$ release, and platelet oxidative status in dyslipidemic patients. Our results showed that argan oil produced an anti-aggregant effect after it had been consumed for three weeks instead of butter at breakfast. This anti-aggregant effect was demonstrated by a $29 \%$ decrease in thrombin-induced aggregation and a tendency towards lower baseline $\mathrm{TxB}_{2}$ levels in the AO group than in controls. In-vitro, an inhibition of thrombin- or epinephrine-induced aggregation by up to $50 \%$ was obtained in isolated rats platelets with a dose of $0.5 \%$ of argan oil [27]. At least two mechanisms are probably involved by which the fatty acids regulate platelet function: modulation of the pool of precursor fatty acids and their competitors, with subsequent effects on eicosanoid metabolism, and changes in cell membrane fluidity, resulting in changes in the activity and/or efficiency of platelet agonist receptors $[54,55]$. The chemical composition of argan oil is dominated by unsaturated fatty acids (80\%), principally oleic and linoleic acids. These unsaturated fatty acids are known to be precursors of prothrombotic and pro-inflammatory eicosanoids; so the anti-aggregant effect observed could be related to the unsaponifiable fraction of argan oil (1\% of the oil constituents), which is richer in tocopherols than olive oil. It also contains other important compounds, such as phenols, principally ferulic and syringic acids, which have already been reported to be anti-aggregants [56]. However, several studies have related the anti-aggregant effect of linoleic acid to the synthesis of prostaglandins $\mathrm{E} 1\left(\mathrm{PGE}_{1}\right)$ and prostacyclins $\mathrm{I}_{2}\left(\mathrm{PGI}_{2}\right)$, which share the same receptor in platelet membranes and inhibit platelet aggregation by increasing cyclic AMP (cAMP) through the activation of receptor-linked adenylate cyclase [57,58]. Furthermore, most oxidant-induced alterations are influenced by the nature of the cellular redox status. It is known that the increased lipid peroxidation observed in some redoxsensitive pathological states (such as dyslipidemia and diabetes) [59] is closely associated with impairment of the antioxidant defenses against peroxides, i.e. decreased activity of GPx [60], the main enzyme that catalyzes the reduction of lipid hydroperoxides to the corresponding alcohols. The results obtained on the platelet oxidative status showed an antioxidant effect of argan oil after three weeks of intervention. This antioxidant effect was manifested by lower platelet MDA levels and higher platelet GPx activity in patients who had consumed argan oil for three weeks compared to control patients who had not. In spite of the high antioxidant content of argan oil, little is known about its mechanism of action. In-vitro investigations of the antioxidant effect of argan oil have reported that incubating LDL with tocopherols, sterols, and phenolic extracts of argan oil significantly prolonged the lagphase of LDL peroxidation [61]. Also, phenolic extracts have been shown to lower the rate of lipid peroxidation, and reduce the disappearance of vitamin $\mathrm{E}$ in a concentration-dependent manner [61]. Other ex-vivo studies have investigated whether the consumption of argan oil could improve antioxidant status in healthy male subjects [23]. Indeed, polyphenols, tocopherols, and sterols could all act as powerful antioxidants via several mechanisms: scavenging of peroxy radicals that break the peroxidation chain reaction; chelating free $\mathrm{Cu}^{2+}$ to form redox-inactive complexes, thus reducing metal-catalyzed oxidation; and inhibiting the binding of $\mathrm{Cu}^{2+}$ to apolipoproteins and subsequently preventing the modification of the amino acid apo-B protein residue [62].

In conclusion, our study shows that argan oil intake for a period of three weeks reduces the risk factors for prothrombotic complications by decreasing plasma cholesterol levels and preventing the platelet hyperactivity and oxidative stress associated with dyslipidemia. Despite the limitations of our study due to the difficulty of collecting sufficient blood to perform all the platelet analysis, these new data are of considerable relevance to the management of dyslipidemia, which is a major risk factor for CVD. Finally, argan oil could be part of the dietary advice provided in the context of the nutritional management of atherosclerotic CVD.

\section{Abbreviations}

CVD: Cardiovascular disease; LDL: Low-density lipoprotein; HDL: High-density lipoprotein; apo-B: Apolipoprotein $\mathrm{B}_{\text {; }}$ TxA $\mathrm{A}_{2}$ : Thromboxane $\mathrm{A}_{2}$;

TBARS: Thiobarbituric acid reactive substances; CPD: Citric phosphate dextrose; BMI: Body mass index; PRP: Platelet-rich plasma;

MDA: Malondialdehyde; GPx: Glutathione peroxidase; IDF: International Diabetes Federation; MS: Metabolic syndrome; SFA: Saturated fatty acids; MUFAs: Monounsaturated fatty acids; PUFAs: Polyunsaturated fatty acids; 
NCEP: National Cholesterol Education Program; GGE $_{1}$ : Prostaglandins E1; $P G I_{2}$ : Prostacyclins $I_{2}$.

\section{Competing interests}

The authors declare that they have no competing interests.

\section{Authors' contributions}

$\mathrm{AH}$ drafted the manuscript and was involved in study design and performed statistical and data analysis. HM carried out sample collection and storage and participated in sample preparation and analysis. LU participated in results discussion and manuscript preparation. VM participated in results discussion and manuscript preparation. AM contributed to the platelet results discussion. AC participated in the coordination of patients recruitment. GT participated in the manuscript preparation. NM participated in the planning and organization of the study, in the experimental work and manuscript preparation. All the authors have read and approved the final manuscript.

\section{Acknowledgements}

The research is supported by an "accord programme PHC-Volubilis $n^{\circ} \mathrm{MA}$ / 21/61 with joint financial support from the French Foreign Affairs Ministry and the Moroccan Ministry of Research and Higher Education. The authors gratefully thank Zakaria Ouissafane for providing the argan oil used in this study. We would also like to thank the doctors and the nursing staff at the Endocrinology, Diabetology and Nutrition Department of the Ibn Sina University Hospital, Rabat, Morocco for their assistance in carrying out physical examinations and collecting blood. Our grateful thanks also to the technical staff of the Hemostasis Laboratory at the Ibn Sina University Hospital, Rabat, Morocco for their warm reception. The authors would also like to thank M. Ghosh for reviewing the English text.

\section{Author details}

'IUT Département Génie Biologique, Université du Maine, PRES L'UNAM, EA 2160 MMS (Mer, Molécules, Santé), Faculté des Sciences et Techniques, Le Mans, Laval, France. ${ }^{2}$ Université Hassan II, Equipe Nutrition, Environnement, Santé. Laboratoire de Virologie, Microbilogie, Qualité/Ecotoxicologie et Biodiversité, Faculté des Sciences et Techniques, Mohammedia, Morocco. ${ }^{3}$ Centre Hospitalier Universitaire Ibn Sina, Service d'Endocrinologie Diabétologie et Nutrition, Rabat, Morocco. ${ }^{4}$ Centre Hospitalier Universitaire Ibn Sina, Laboratoire d'Hémostase, Faculté de Médecine et de Pharmacie, Rabat, Morocco.

Received: 15 May 2013 Accepted: 4 July 2013

Published: 20 July 2013

\section{References}

1. Anonymous: Santé en chiffres 2011. Maroc: Ministère de la santé; 2012:5-180.

2. Grundy SM: Metabolic syndrome: a multiplex cardiovascular risk factor. J Clin Endocrinol Metab 2007, 92:399-404.

3. Jacobson TA, Miller M, Schaefer EJ: Hypertriglyceridemia and cardiovascular risk reduction. Clin Ther 2007, 29:763-777.

4. Lacoste L, Lam JYT, Hang J, Letchacovski G, Solymoss CB, Waters D: Hyperlipidemia and coronary disease. Circulation 1995, 92:3172-3177.

5. McNeal C, Wilson DP: Metabolic syndrome and dyslipidemia in youth. J Clin Lipidol 2008, 2:147-155.

6. Serebruany VL, Malinin A, Ong S, Atar D: Patients with metabolic syndrome exhibit higher platelet activity than those with conventional risk factors for vascular disease. J Thromb Thrombolysis 2008, 25:207-213.

7. Alexandru N, Popov D, Georgescu A: Platelet dysfunction in vascular pathologies and how can it be treated. Thromb Res 2012, 129:116-126.

8. Badimón L, Vilahur G, Padró T: Lipoproteins, platelets, and atherothrombosis. Rev Esp Cardiol 2009, 62:1161-1178.

9. Huo Y, Ley KF: Role of the platelets in the development of atherosclerosis. Trends Cardiovasc Med 2004, 14:18-22.

10. Libby P. Inflammation in atherosclerosis. Nature 2002, 420:868-874.

11. David G, Giammarresi C, Barbagallo C, Catalano I, Cala A, Notarbartolo A: Thromboxane biosynthesis, neutrophil and coagulative activation in type lla hypercholesterolemia. Thromb Haemost 1995, 74:1015-1019.

12. Shi G, Morrell CN: Platelets as initiators and mediators of inflammation at the vessel wall. Thromb Res 2011, 127:387-390.
13. Bhupathiraju SN, Tucker KL: Coronary heart disease prevention: Nutrients, foods and dietary patterns. Clin Chim Acta 2012, 412:1493-1514.

14. Mitjavila MT, Fandos M, Salas-Salvadós J, et al: The Mediterranean diet improves the systemic lipid and DNA oxidative damage in metabolic syndrome individuals. A randomized controlled trial. Clin Nutr 2013, 32:172-178.

15. Erkkilä A, De Mello VDF, Risérus U, Laaksonen DE: Dietary fatty acids and cardiovascular disease: An epidemiological approach. Prog Lipid Res 2008, 47:172-187.

16. Haimeur A, Ulmann L, Mimouni V, Guéno F, Pineau-Vincent F, Meskini $N$, Tremblin G: The role of Odontella aurita, a marine diatom rich in EPA, as a dietary supplement in dyslipidemia, platelet function and oxidative stress in high-fat fed rats. Lipids Health Dis 2012, 11:147.

17. Charrouf Z, Guillaume D: Ethnoeconomical, ethnomedical and phytochemical study of Argania spinosa (L) skeels. J Ethnopharmacol 1999, 67:7-14.

18. Khallouki F, Younos C, Soulimani R, et al: Consumption of argan oil (Morocco) with its unique profile of fatty acids tocopherols squalene sterols and phenolic compounds should confer valuable cancer chemopreventive effects. Eur J Canc Prev 2003, 12:67-75.

19. Bellahcen S, Mekhfi H, Ziyyat $\mathrm{A}$, et al: Prevention of chemically induced diabetes milletus in experimental animals by virgin argan oil. Phytother Res 2012, 26:180-185.

20. Berrougui H, Ettaib A, Herrena Gonzalez MD, Alvez De Sotomayor M, Bennani Kabchi N, Hmamouchi M: Hypolipidemic and hypocholesterolemic effect of argan oil (Argania spinosa L.) in Meriones shawi rats. J Ethnopharmacol 2003, 89:15-18.

21. Marhuenda E, Herrera MD: Argan (Argania spinosa) oil lowers blood pressure and improves endothelial dysfunction in spontaneously hypertensive rats. Br J Nut 2004, 92:921-929.

22. Samane $S$, Christon R, Dombrowski $L$, et al: Fish oil and argan oil intake differently modulate insulin resistance and glucose intolerance in a rat model of dietary-induced obesity. Metab Clin Exp 2009, 58:909-919.

23. Cherki M, Derouiche A, Drissi A, et al: Consumption of argan oil may have an antiatherogenic effect by improving paraoxonase activities and antioxidant status: Intervention study in healthy men. Nutr Metab Cardiovasc Dis 2005, 15:352-360.

24. Drissi A, Girona J, Cherki M, et al: Evidence of hypolipemiant and antioxidant properties of argan oil derived from the argan tree (Argania spinosa). Clin Nutr 2004, 23:1159-1166.

25. Derouiche A, Cherki M, Drissi A, et al: Nutritional intervention study with argan oil in man: effects on lipids and apolipoproteins. Ann Nutr Metab 2005, 49:196-201.

26. Samane S, Noël J, Charrouf Z, Amarouch H, Haddad PS: Insulin-sensitizing and anti-proliferative effects of Argania spinosa seed extracts. Evid Based Complement Alternat Med 2006, 3:317-327.

27. Mekhfi H, Gadi D, Bnouham M, Ziyyat A, Legssyer A, Aziz M: Effect of argan oil on platelet aggregation and bleeding time: a beneficial nutritional property. J Compl Integr Med 2008, 5:1-18.

28. Mekhfi H, Belmekki F, Ziyyat A, Legssyer A, Bnouham M, Aziz M: Antithrombotic activity of argan oil: An in vivo experimental study. Nutrition 2012, 28:937-941.

29. Reiner Z, De Backer G, Graham I, et al: ESC/EAS Guidelines for the management of dyslipidemias The task force for the management of dyslipidemias of the European Society of Cardiology (ESC) and the European Atherosclerosis Society (EAS). Atherosclerosis 2011, 217:3-46.

30. Boukhobza M, Pichon-Prun N: L'arganier ressource économique et médicinale pour le Maroc. Phytothérapie 1988, 27:21-26.

31. Richmond W: Preparation and properties of a cholesterol oxidase from Nocardia sp. and its application to the enzymatic assay of total cholesterol in serum. Clin Chem 1973, 19:1350-1356

32. Trinder P: Enzymatic method of triglycerides. Ann Clin Biochem $1969,6: 24-27$.

33. Friedwald WT, Levy RI, Fredrickson DS: Estimation of the concentration of low-density lipoprotein cholesterol in plasma without use of the preparative ultracentrifuge. Clin Chem 1972, 18:499-502

34. Lagarde M, Bryon PA, Guichardant M, Dechavanne MA: Simple and efficient method for platelet isolation from their plasma. Thromb Res 1980, 17:581-588.

35. Born GVR: Aggregation of blood platelets by adenosine diphosphate and its reversal. Nature 1962, 194:927-929. 
36. Ohkawa H, Ohishi N, Yagik K: Assay for lipid peroxides in animal tissues by thiobarbituric acid reaction. Anal Biochem 1979, 95:351-358.

37. Paglia DE, Valentine WN: Studies on the quantitative and qualitative characterization of erythrocyte glutathione peroxidase. J Lab Clin Med 1967, 70:158-169.

38. Chaudiére J, Gerard D: Dosage de l'activité glutathion-peroxydase. In Biologie des Lipides chez l'Homme. Edited by Douste-Blazy L, Mendy F. Paris: Médicales Internationales; 1988:275-289.

39. Bradford MM: A rapid and sensitive method for the quantitation of microgram quantities of protein utilizing the principle of protein- dye binding. Anal Biochem 1976, 72:248-254.

40. Alberti KG, Zimmet $P$, Shaw J: The metabolic syndrome-a new worldwide definition. Lancet 2005, 366:1059-1062.

41. Berrougui $\mathrm{H}$, Cherki M, Koumbadinga GA, et al: Antiatherogenic activity of extracts of Argania spinosa L. pericarp: beneficial effects on lipid peroxidation and cholesterol homeostasis. Can J Physiol Pharmacol 2007, 85:918-927.

42. Cherki M, Berrougui H, Drissi A, Adlouni A, Khalil A: Argan oil: which benefits on cardiovascular diseases? Pharmacol Res 2006, 54:1-5.

43. Elmanfalouti H, Guillaume D, Denhez C, Charrouf Z: Therapeutic potential of argan oil: a review. J Pharm Pharmacol 2010, 62:1669-1675.

44. Kris-Etherton P, Pearson TA, et al: High monounsaturated fatty acid diets lower both plasma cholesterol and triacyglycerol concentrations. Am J Clin Nutr 1999, 70:1009-1015.

45. Williams CM, Francis-Knapper JA, Webb D, et al: Cholesterol reduction using manufactured foods high in monounsaturated fatty acids: a randomized crossover study. Br J Nutr 1999, 81:439-446.

46. Perez-Jimenez $\mathrm{F}$ : International conference on the healthy effect of virgin olive oil. Eur J Clin Invest 2005, 35:421-424.

47. Nijjar PS, Burke FM, Bloesch A, Rader DJ: Role of dietary supplements in lowering low-density lipoprotein cholesterol: A review. J Clin Lipidol 2010, 4:248-258.

48. Takada R, Saitoh M, Mori T: Dietary gamma-linolenic acid-enriched oil reduces body fat content and induces liver enzyme activities relating to fatty acid beta-oxidation in rats. J Nutr 1994, 124:469-474.

49. Patch CS, Tapsell LC, Williams PG, Gordon M: Plant sterols as dietary adjuvants in the reduction of cardiovascular risk: theory and evidence. J Vasc Health Risk Manag 2006, 2:157-162.

50. Calpe-Berdiel L, Escolà-gil JC, Blanco-Vaca F: New insights into the molecular actions of plant sterols and stanols in cholesterol metabolism. Atherosclerosis 2009, 203:18-31.

51. Jones PI, Raeini-Sarjaz M, Ntanios FY, Vanstone CA, Feng JY, Parsons WE: Modulation of plasma lipid levels and cholesterol kinetics by phytosterol versus phytostanol esters. J Lipid Res 2000, 41:697-705.

52. Maki KC, Lawless AL, Reeves MS, et al: Lipid effects of a dietary supplement softgel capsule containing plant sterols/stanols in primary hypercholesterolemia. Nutrition 2013, 29:96-100.

53. Rice-Evans C, Burdon C: Free radical-lipid interactions and their pathological consequences. Prog Lipid Res 1993, 32:71-110.

54. Das UN: Essential fatty acid metabolism in patients with essential hypertension diabetes mellitus and coronary heart diseases. Prostaglandins Leukot Essent Fatty Acids 1995, 52:387-391.

55. Hornstra G, Rand ML: Influence of dietary lipids on platelet-vessel wall interaction: possible role of eicosanoids and platelet membrane fluidity. Haemostasis 1986, 16:41-42.

56. Ostertag LM, O'Kennedy N, Horgan GW, Kroon PA, Duthie GG, De Roos B: In vitro anti-platelet effects of simple plant-derived phenolic compounds are only found at high non-physiological concentrations. Mol Nutr Food Res 2011, 55:1624-1636.

57. Burri BJ, Dougherty RM, Kelley DS, lacono M: Platelet aggregation in humans is affected by replacement of dietary linoleic acid with oleic acid. Am J Clin Nutr 1991, 54:359-362.

58. Dutta-Roy AK, Sinha AK: Purification and properties of prostaglandin $E_{1} /$ prostacyclin receptor of human blood platelets. J Biol Chem 1987, 262:12685-12691.

59. Nourooz-Zadeh J, Tajaddini-Sarmadi J, McCarthy S, Betteridge DJ, Wolff SP: Elevated levels of authentic plasma hydroperoxides in NIDDM. Diabetes 1995, 44:1054-1058.

60. Muruganandam A, Drouillard C, Thibert RJ, Cheung RM, Draisey TF, Mutus B: Glutathione metabolic enzyme activities in diabetic platelets as a function of glycemic control. Thromb Res 1992, 67:385-397.
61. Berrougui $H$, Cloutier M, Isabelle M, Khalil A: Phenolic-extract from argan oil (Argania spinosa L.) inhibits human low-density lipoprotein (LDL) oxidation and enhances cholesterol efflux from human THP-1 macrophages. Atherosclerosis 2006, 184:389-396.

62. Jialal I, Fuller CJ, Huet BA: The effect of a-tocopherol supplementation on LDL oxidation a dose response study. Arterioscler Thromb Vasc Biol 1995, 15:190-198.

doi:10.1186/1476-511X-12-107

Cite this article as: Haimeur et al:: Argan oil prevents prothrombotic complications by lowering lipid levels and platelet aggregation, enhancing oxidative status in dyslipidemic patients from the area of Rabat (Morocco). Lipids in Health and Disease 2013 12:107.

\section{Submit your next manuscript to BioMed Central and take full advantage of:}

- Convenient online submission

- Thorough peer review

- No space constraints or color figure charges

- Immediate publication on acceptance

- Inclusion in PubMed, CAS, Scopus and Google Scholar

- Research which is freely available for redistribution 